\title{
Five new Palaearctic species of Docosia (Diptera: Mycetophilidae), with updated molecular phylogeny of the genus
}

\author{
Jan ŠEVČÍK ${ }^{1, *}$, Nikola BURDÍKOVÁ ${ }^{2}$, David KASPŘÁK ${ }^{3}$ \& Olavi KURINA ${ }^{4}$ \\ ${ }^{1,2,3}$ University of Ostrava, Faculty of Science, Department of Biology and Ecology, \\ Chittussiho 10, CZ-710 00 Ostrava, Czech Republic. \\ ${ }^{1}$ Silesian Museum, Nádražní okruh 31, CZ-746 01 Opava, Czech Republic. \\ ${ }^{4}$ Institute of Agricultural and Environmental Sciences, Estonian University of Life Sciences, \\ Kreutzwaldi st 5D, 51006 Tartu, Estonia. \\ *Corresponding author: sevcikjan@hotmail.com \\ 2Email: burdikova@seznam.cz \\ ${ }^{3}$ Email: davidkasprak@gmail.com \\ ${ }^{4}$ Email: olavi.kurina@emu.ee

\footnotetext{
${ }^{1}$ urn:lsid:zoobank.org:author:639373EF-6D46-40F6-929B-0C861C6C39F6

${ }^{2}$ urn:lsid:zoobank.org:author:F7CCFBF7-CB4E-489A-95AC-7346268EB0E0

${ }^{3}$ urn:1sid:zoobank.org:author:665C08DD-1099-4F88-B528-A9441AFC6A36

${ }^{4}$ urn:lsid:zoobank.org:author:FB595938-73A2-4DBC-9ABB-77E81D13DFE1
}

\begin{abstract}
A new phylogenetic hypothesis is proposed for the relationships among the species within the genus Docosia Winnertz, 1863, based on a combined analysis of five DNA markers (28S, ITS2, COI, COII and CytB). Five new species are described, Docosia anatolica Ševčík sp. nov. from Turkey, D. japonica Kurina sp. nov. from Japan, D. peloponnensis Ševčík sp. nov. from Greece, D. svanetica Kurina sp. nov. from Caucasus and D. polyspina Kurina sp. nov. from the Russian Far East. New country records of the following species are presented: D. diutina Plassmann, 1996 (Turkey), D. flavicoxa Strobl, 1900 (Georgia), D. gilvipes (Haliday in Walker, 1856) (Georgia), D. kerkini Kurina \& Ševčík, 2011 (Bulgaria), D. moravica Landrock, 1916 (Georgia), D. pannonica Lastovka \& Ševčík 2006 (Georgia) and D. rameli Kurina \& Ševč́́k, 2011 (Slovakia).
\end{abstract}

Keywords. Bibionomorpha, Sciaroidea, fungus gnats, taxonomy, DNA sequences.

Ševčík J., Burdíková N., Kasprák D. \& Kurina O. 2020. Five new Palaearctic species of Docosia (Diptera: Mycetophilidae), with updated molecular phylogeny of the genus. European Journal of Taxonomy 717: 3-26. https://doi.org/10.5852/ejt.2020.717.1095

\section{Introduction}

Fungus gnats of the genus Docosia Winnertz, 1863 represent a rather well-defined group within the family Mycetophilidae Newman, 1834 (Diptera Linnaeus, 1758), with a mostly dark body, unmarked wings (Fig. 1) and identification characters present mainly on the male terminalia (Laštovka \& Ševčík 
2006). It has been demonstrated that characters used to separate species in the keys, other than on male terminalia, do not correspond to the relationships revealed by molecular methods (Ševčík et al. 2016a). According to recent molecular studies, the genus Docosia belongs to the subfamily Gnoristinae Edwards, 1925, although this subfamily appears as paraphyletic, with respect to Mycetophilinae (Rindal et al. 2009; Ševčík et al. 2013; Kaspř́k et al. 2019).

The biology of species of Docosia is mostly unknown (Chandler 2010; Ševčík 2010a; Jakovlev 2012), except for Docosia fumosa Edwards, 1925 and Docosia gilvipes (Haliday in Walker, 1856), which are known to develop in nests of birds (Rulik \& Kallweit 2006) and fungi, respectively.

The known distribution of Docosia includes the Holarctic Region, with three species reported from the Neotropical Region (Oliveira \& Amorim 2011, 2014) and a single species from the Oriental Region (Ševčík 2010b). The common European species Docosia gilvipes was also recorded from the Republic of South Africa (Kurina \& Ševčík 2012). Altogether, 77 extant species have been described so far from the Holarctic Region including 57 from the Palaearctic and 20 from the Nearctic Regions (reviewed by Zaitzev 2011; Kurina \& Ševčík 2012; Taber 2012; 2018; Ševčík et al. 2016a). Within the Palaearctic Region, besides the relatively well-studied Europe with 34 known species (Kurina \& Ševčík 2012; Ševčík et al. 2016a), Central Asia has 11 species recorded (Kurina 2006; Zaitzev 2011; Kurina \& Ševčík 2012), while the other areas are rather sporadically sampled and studied.

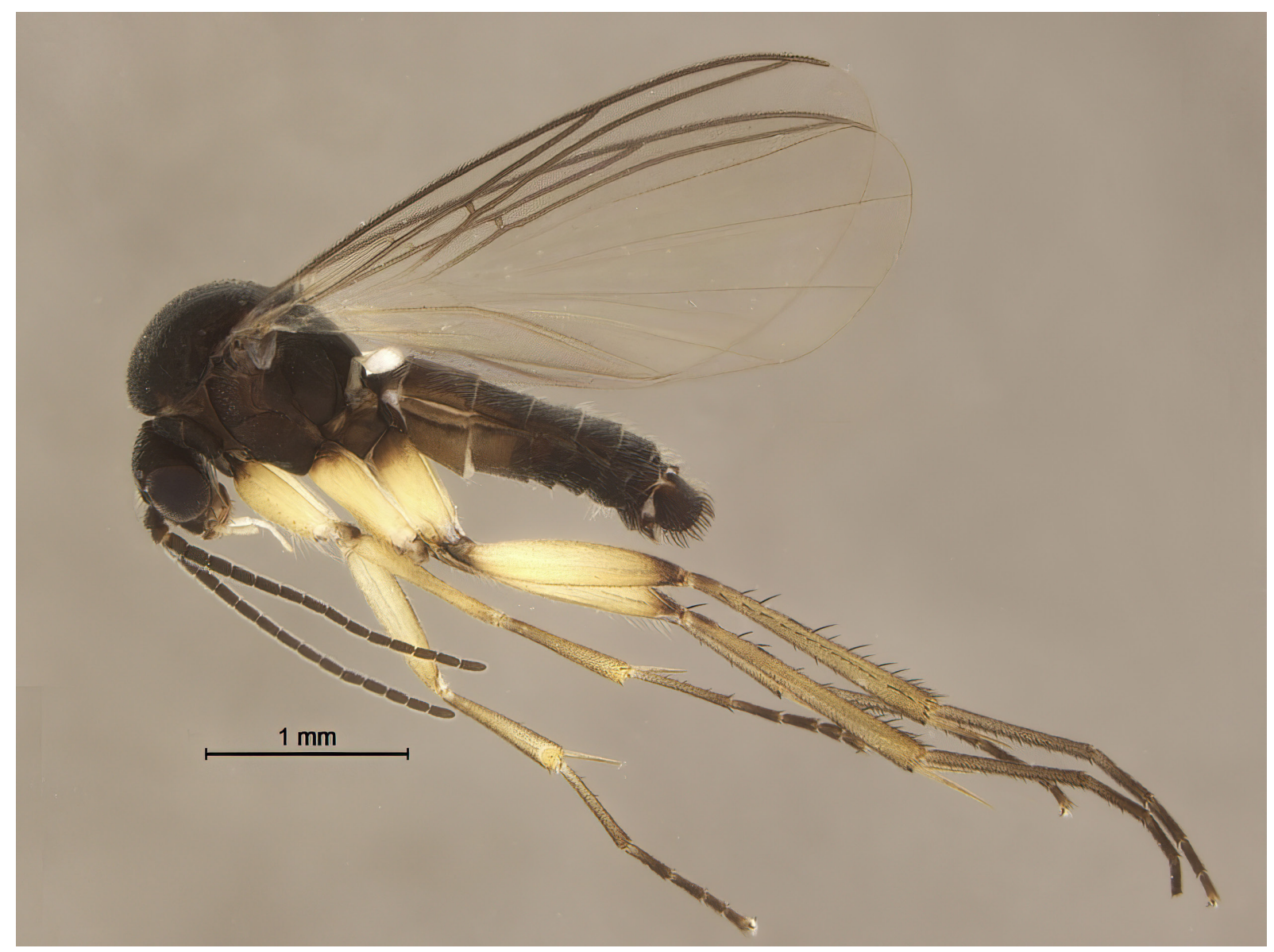

Fig. 1. Docosia svanetica Kurina sp. nov., paratype (ZFMK-DIP-00067331), habitus. 
The phylogenetic relationships among particular species of Docosia are still poorly known, as well as the species spectrum in less-studied areas. The first attempt to reconstruct the phylogeny of several Central European species of Docosia was that by Ševčík et al. (2016a), based on three mitochondrial gene fragments and the nuclear ITS2 region. Here, we present a new hypothesis for the relationships among the species of Docosia, which is a result of an expanded dataset, also including the nuclear $28 \mathrm{~S}$ gene region, and with more than twice as many species of Docosia included.

The opportunity is also taken here to describe five new Palaearctic species, updating the number of Palaearctic species of Docosia to 62 (Ševčík \& Laštovka 2008; Kurina \& Ševčík 2011; Ševčík et al. 2016a), and to present new national records of several rare species.

Morphological terminology principally follows that of Søli (1997, 2017).

\section{Material and methods}

The material used for DNA extraction and species descriptions was collected with Malaise traps, light traps or sweep nets at various localities in the years 2012-2019 (see Table 1). The voucher specimens are deposited in the following depositories:

IUTG $=$ Collection of Ilia State University, Tbilisi, Georgia

IZBE $=$ Institute of Agricultural and Environmental Sciences, Estonian University of Life Sciences (former Institute of Zoology and Botany), Tartu, Estonia

JSL-OUC $=$ Collection of Jan Ševčík Lab, University of Ostrava, Czech Republic

NMPC $=$ National Museum, Prague, Czech Republic

ZFMK $=$ Zoological Research Museum Alexander Koenig, Bonn, Germany

Most of the specimens were collected into $70 \%$ ethanol. The type specimens are stored in glycerine medium in a plastic pinned microvial, in ethyl alcohol, or mounted from alcohol, using the chemical method described by Vockeroth (1966), and double-mounted using minute pins. For every studied specimen, the preservation method is indicated in the material section. Illustrations of the terminalia were prepared using a U-DA drawing tube attached to a compound microscope Olympus CX31. The digital images of the general habitus and terminalia were combined using the software LAS ver. 4.1.0. from multiple gradually focused images taken by a Leica DFC 450 camera attached to a Leica 205C stereo microscope or a Leica DM 6000 B compound microscope, respectively (see also Jürgenstein et al. 2015). Adobe Photoshop CS5 was used for editing the figures and compiling the plates. Before placing in glycerol, the specimens of several species were treated in proteinase K to extract DNA. The protocol of DNA extraction, amplification, sequencing and phylogenetic analysis is described in Ševčík et al. (2016b). The primers used for the PCR amplifications of mitochondrial and nuclear gene fragments are listed in Ševčík et al. (2016a, 2016b).

The final molecular dataset consists of 3049 characters: $28 \mathrm{~S}-924 \mathrm{bp}$, ITS2 - $488 \mathrm{bp}, \mathrm{COI}-658 \mathrm{bp}$, COII $-546 \mathrm{bp}$, cytB $-433 \mathrm{bp}$. All the sequences were deposited in GenBank (see Table 1). The maximum likelihood (ML) analyses of single gene alignments were conducted on the CIPRES computer cluster using RAxML-HPC BlackBox 8.2.10 (Stamatakis 2014) to check potential conflicting topologies and other artefacts. The final concatenated dataset was partitioned by gene and codon position and subsequently analysed using ML method with IQtree (Nguyen et al. 2015) on the IQ-TREE web server http://iqtree.cibiv.univie.ac.at/ (Trifinopoulos et al. 2016). Best-fitting substitution models were chosen automatically by the IQ-TREE software $(28 \mathrm{~S}-\mathrm{HKY}+\mathrm{F}+\mathrm{I}$; ITS $2-\mathrm{TPM} 2 \mathrm{u}+\mathrm{F}+\mathrm{I}+\mathrm{G} 4$; COI_1 - HKY +F+G4; COI_2 - TIM2+F+G4; COI_3 - F81+F+I; COII_1 - HKY+F+I+G4; COII_2 TIM2+F+G4; COII_3 - TPM2+F+I+G4; cytB_1 - TPM3u+F+G4; cytB_2 - TIM3+F+G4; cytB_3 $\mathrm{TN}+\mathrm{F}+\mathrm{I}+\mathrm{G} 4)$, without free-rate heterogeneity. Branch supports were evaluated using 1000 ultrafast 


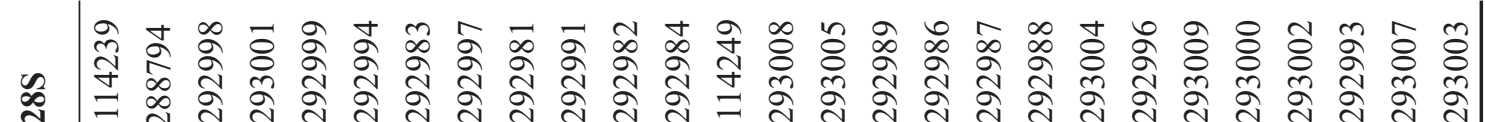

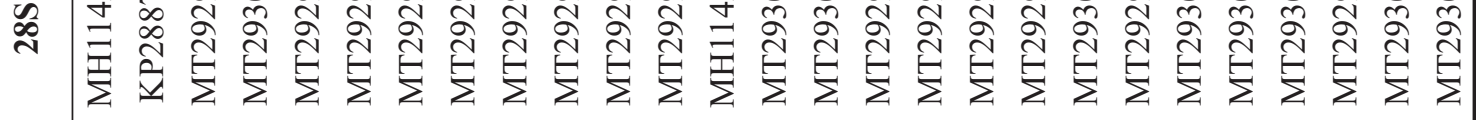

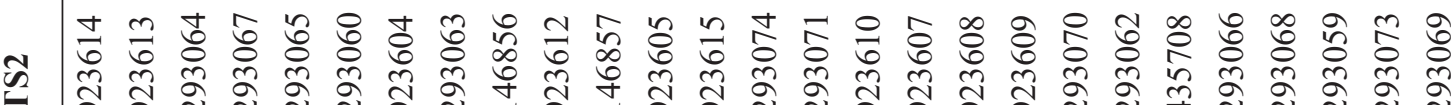

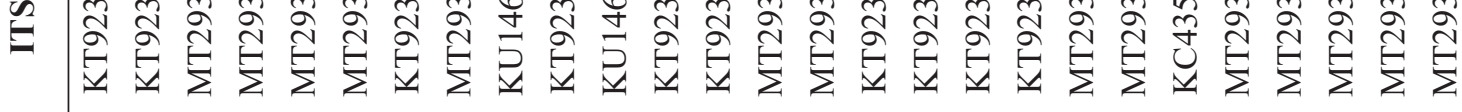

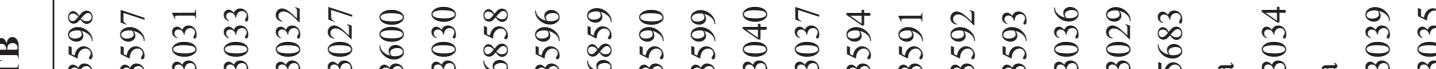

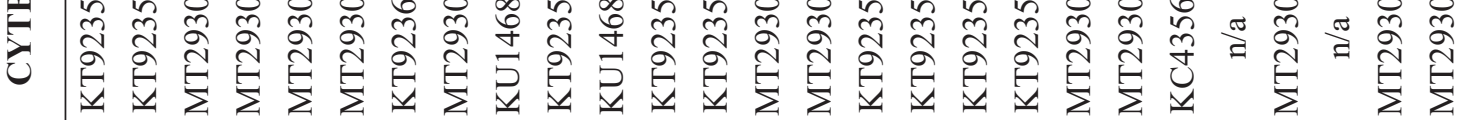

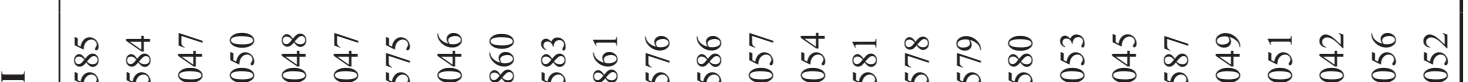

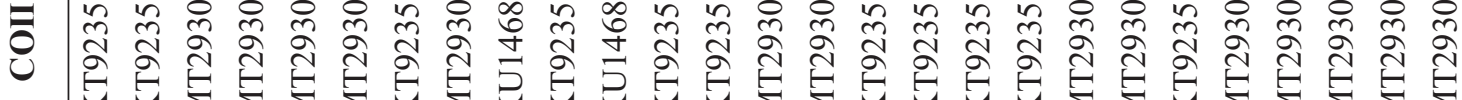

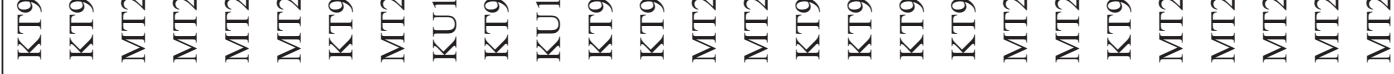

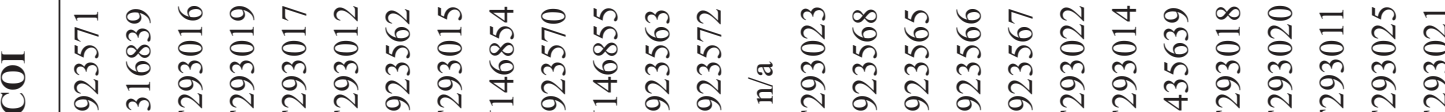

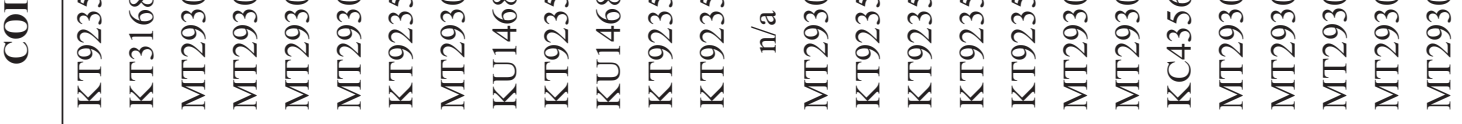

$\frac{\sqrt{n}}{\frac{\pi}{\pi}}$

:

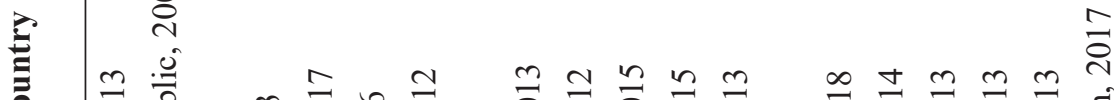

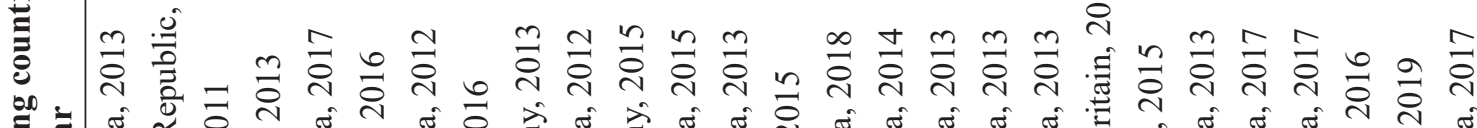

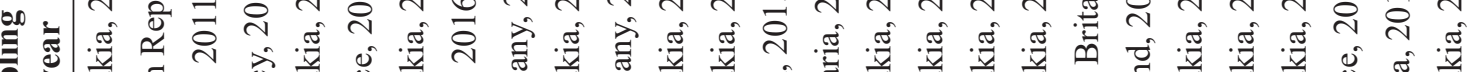

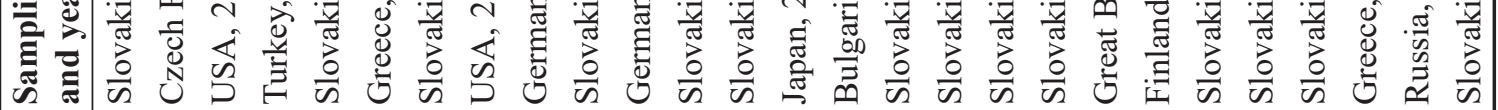<smiles>Cc1ccccc1CSc1ccccn1</smiles>

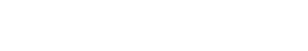

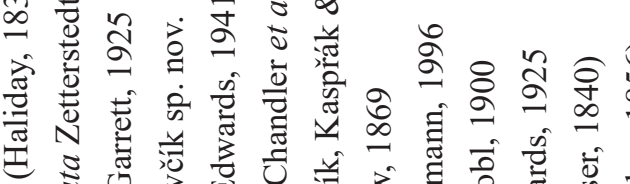

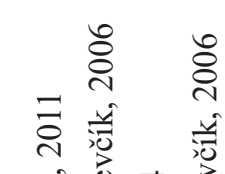

$$
\text { (1) }
$$
ฐ 


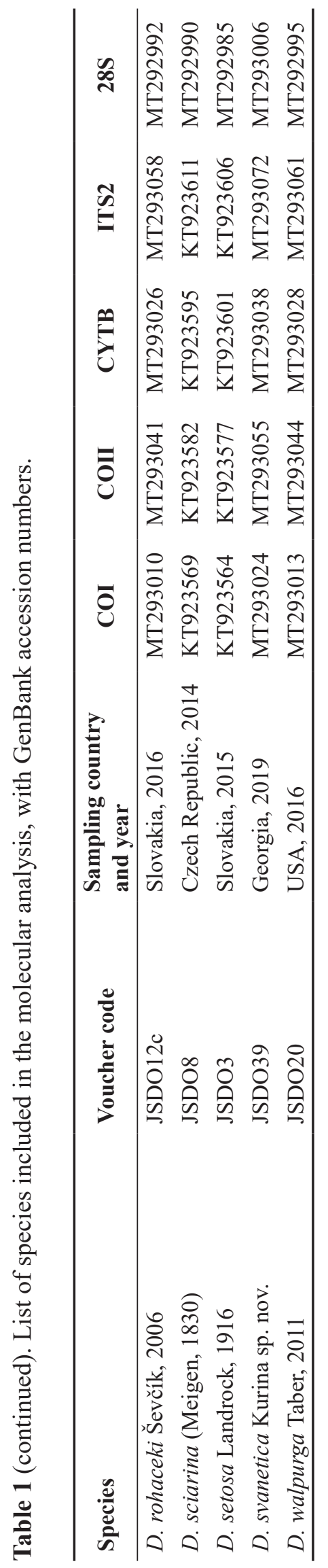


bootstrap (Hoang et al. 2017). All other settings were left as default. The node support values are given in the form of ultrafast bootstrap (= ufboot). Ultrafast bootstrap values are more unbiased - ufboot of 95 corresponds roughly to a probability of $95 \%$ that a clade is true (Minh et al. 2013; Hoang et al. 2017). As outgroup taxa we selected two representatives of the subfamily Gnoristinae, in concordance with the most recent molecular study (Kaspř́k et al. 2019). The resulting phylogenetic tree (consensus tree) was visualized using Interactive Tree Of Life (iTOL; Letunic \& Bork 2016).

\section{Results}

\section{Taxonomic treatments}

Phylum Arthropoda Latreille, 1829

Class Insecta Linnaeus, 1758

Order Diptera Linnaeus, 1758

Infraorder Bibionomorpha Hennig, 1954

Superfamily Sciaroidea Billberg, 1820

Family Mycetophilidae Newman, 1834

Subfamily Gnoristinae Edwards, 1925

Genus Docosia Winnertz, 1863

Docosia anatolica Ševčík sp. nov. urn:Isid:zoobank.org:act:7E7CA4EE-F77C-4B6F-87FA-A42B0F0B5A35

Figs 2, 7A

\section{Differential diagnosis}

By structure of the male terminalia, D. anatolica sp. nov. resembles D. juxtamontana Chandler, 2004 and D. incolamontis Chandler, 2004. All species have a bifid gonostylus and posteroventral margin of gonocoxites with a prominent medial process surrounded by two small lobes bearing setae. These setae are apically ramified in $D$. anatolica sp. nov., but with an unknown structure in the other two species. Apically modified setae at the posteroventral margin of gonocoxites are frequent in CentralAsian species (cf. Kurina \& Ševčík 2012) but they are also observable in other species, e.g., in the European D. pannonica Laštovka \& Ševčík, 2006. Docosia anatolica sp. nov. has the ventral lobe of the gonostylus obtriangular, with spines at both posterior corners, while it is slender and furcated, with medial and apical spines in D. juxtamonta and irregularly crescent-shaped with two subapical spines in D. incolamontis.

\section{Etymology}

The species name refers to the type locality in Anatolia (Asia Minor).

\section{Type material}

\section{Holotype}

TURKEY • O’; Mugla, University Campus; 1100 m a.s.1.; 22 Apr. 2016; M. Barták and Š. Kubík leg.; Malaise trap; in a pinned microvial with glycerol; specimen after DNA extraction; GenBank: MT293019, MT293050, MT293033, MT293067, MT293001; NMPC-JSDO26.

\section{Description}

Male $(\mathrm{n}=1)$

MEASUREMENTs. Length of wing $4.2 \mathrm{~mm}$. 


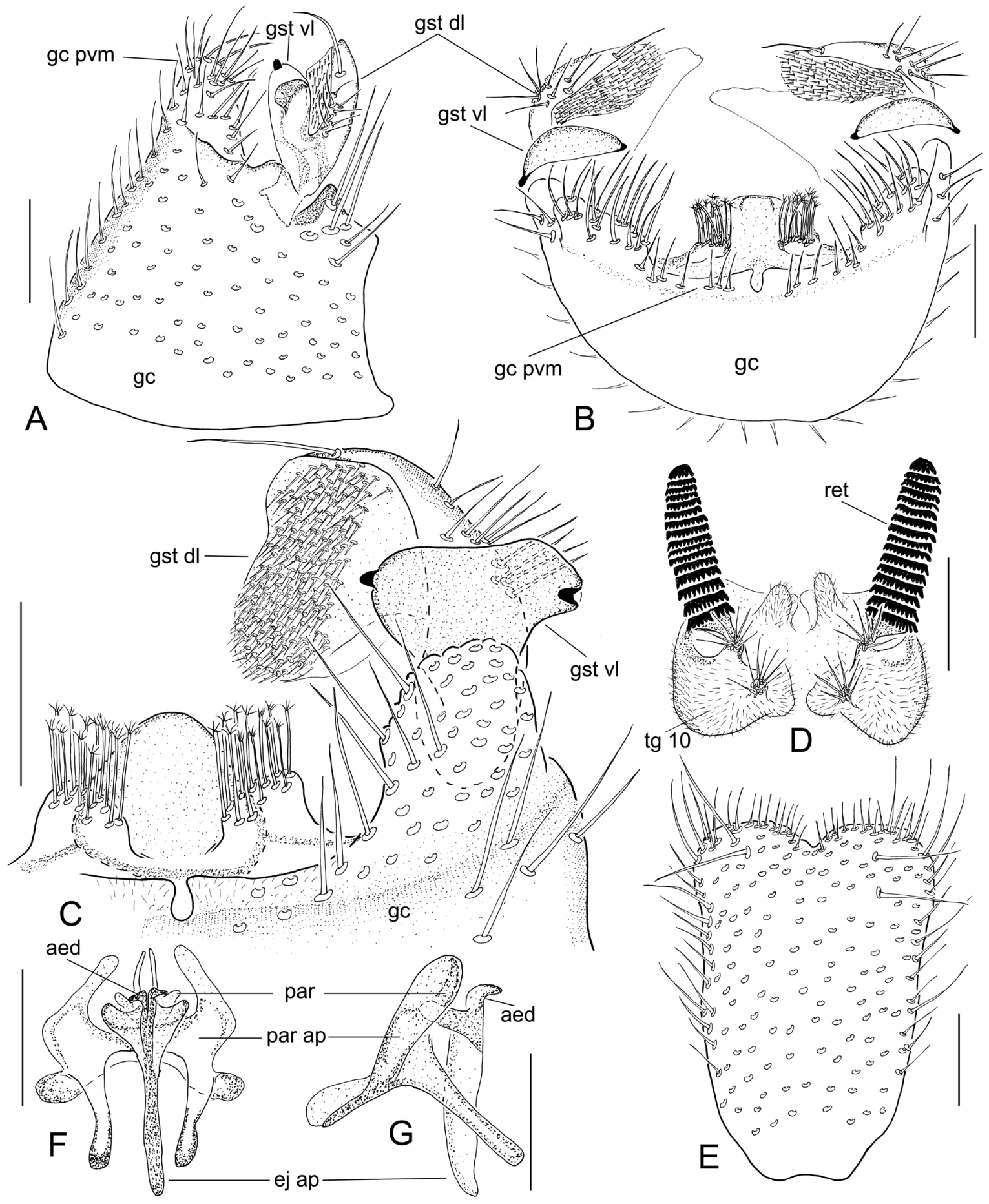

Fig. 2. Docosia anatolica Ševčík sp. nov., ô, terminalia. A. Lateral view. B. Posterior view. C. Ventral view of gonostylus. D. Dorsal view of cerci. E. Dorsal view of tergite 9. F. Ventral view of aedeagal complex. G. Lateral view of aedeagal complex. Abbreviations: aed $=$ aedeagus; ej ap $=$ ejaculatory apodeme; gc $=$ gonocoxite; $\mathrm{gc}$ pvm $=$ posteroventral margin of gonocoxite; gst $\mathrm{vl}=$ ventral lobe of gonostylus; gst $\mathrm{dl}=$ dorsal lobe of gonostylus; par = paramere; par ap = parameral apodeme; ret $=$ combs of retinacula; $\operatorname{tg}=$ tergite. Scale bars $=0.1 \mathrm{~mm}$. 
HEAD. Blackish brown with numerous pale setae. Three ocelli, lateral ones almost touching compound eyes, separated from eye margins by less than half of their own diameter. Mouthparts light brownish. Palpus brownish yellow, basal segment and apical part of last palpomere darker. Scape, pedicel and all flagellomeres dark brown. Flagellomeres cylindrical, flagellomeres 1 and 2 about twice as long as broad, other flagellomeres ( 3 to 14 ) about three times as long as broad.

THORAX. All parts of thorax blackish brown, with light setae. Scutellum with numerous setae and several submarginal pale bristles about twice as long as scutellum. Antepronotum and proepisternum with pale bristles and short darker setae. Laterotergite and other pleural parts bare. Haltere pale yellow.

LEGS. All coxae yellow, with basal third darkened. Femora mostly yellow, fore and mid femur dark along ventral margin, more extensively in proximal third; similarly hind femur, which is brownish also around tip. Trochanters blackish brown. Fore tibia brownish yellow, apicomedially with simple semicircular tibial organ (anteroapical depressed area), without strong setae, only densely covered with fine setulae. Mid tibia darkened dorsally. Hind tibia mostly brownish yellow, with small black dot approximately in middle of dorsal surface.

WINGS. Hyaline, unmarked. Radial veins and r-m dark brown, other veins paler, basal parts of $m$ faint, almost not traceable. Sc, Rs and basal third of cu-stem asetose, other veins setose. Costa reaches to about one third of distance between $\mathrm{R}_{5}$ and $\mathrm{M}_{1}$. Sc ends in $\mathrm{R}$ before level of beginning of $\mathrm{m}$-stem. Posterior fork begins before anterior fork, approximately at level of basal third of r-m.

ABDOMEN. All dark brown.

Terminalia (Figs 2A-E, 7A). Dark brown except lighter gonostyli and posterior margin of gonocoxites. Tergite 9 subrectangular, about 1.5 times as long as broad, slightly broadening posteriorly, posterior margin with medial incision. Posteroventral margin of gonocoxites with blunt lateral projections and prominent rounded process medioventrally, surrounded by two shorter processes bearing apically ramified thick setae. Gonostylus bifid. Ventral lobe of gonostylus obtriangular in ventral view, with dark tooth at both posterior corners. Dorsal lobe of gonostylus larger than ventral lobe, bent inwards and rounded apically, with large aggregation of setulae along ventral surface anteriorly, prominent subapical seta posteriorly and with about 10 setae posterolaterally. Cercus with 16 combs of retinacula.

\title{
Female
}

Unknown.

Biology

Unknown.

\author{
Docosia japonica Kurina sp. nov. \\ urn:lsid:zoobank.org:act:8D2164B1-0649-41BD-A65A-28434E54E1F4 \\ Figs 3, 7B
}

\section{Differential diagnosis}

By the structure of the male terminalia, D. japonica sp. nov. belongs to a group of Palaearctic species, as defined for D. polyspina Kurina sp. nov., and resembles D. kerkini Kurina \& Ševčík, 2011, D. polyspina sp. nov. and $D$. svanetica Kurina sp. nov. All species have a simple sickle- or crescent-shaped gonostylus with a row of spines on the medial margin. However, unlike the other species, D. japonica sp. nov. has four medially situated spines with the posteriormost one three times as long as the anterior three (D. kerkini has 12 subequal spines, D. polyspina sp. nov. has 3 subequal spines and $D$. svanetica sp. nov. 


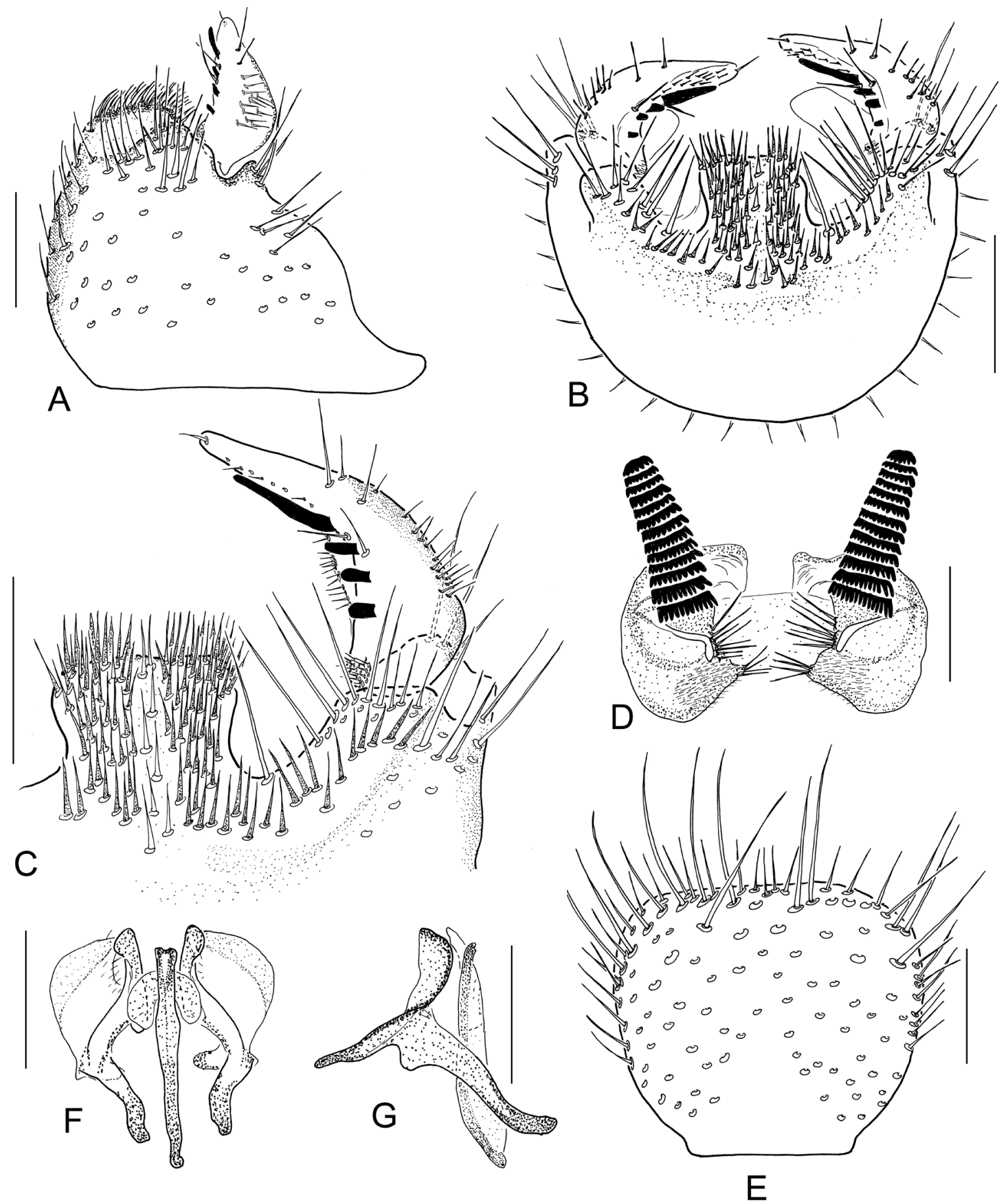

Fig. 3. Docosia japonica Kurina sp. nov., đ̂, terminalia. A. Lateral view. B. Posterior view. C. Ventral view of gonostylus. D. Dorsal view of cerci. E. Dorsal view of tergite 9. F. Ventral view of aedeagal complex. G. Lateral view of aedeagal complex. Scale bars $=0.1 \mathrm{~mm}$. 
has 5 spines with the anteriormost one at the apex of the gonostylus and two times as long as the other). The posteroventral margin of the gonocoxites resembles somewhat D. selini Kurina, 2006 described from Kazakhstan and subsequently found in the Chimgan area in Uzbekistan, but both species can be well distinguished by the shape of the gonostylus.

\section{Etymology}

The species is named after its occurrence in Japan.

\section{Type material}

\section{Holotype}

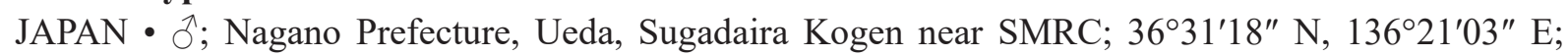
1330 m a.s.1.; 26 Apr.-3 May 2015; A. Blanke leg.; Malaise trap in grassland; in ethyl alcohol; terminalia in glycerol; one fore leg used for DNA extraction; GenBank: MT293057, MT293040, MT293074, MT293008; ZFMK-DIP-00067333.

\section{Description}

$$
\text { Male }(n=1)
$$

MeASuREMENTs. Length of wing $3.8 \mathrm{~mm}$.

HEAD. Black with numerous pale setae. Three ocelli, lateral ones almost touching compound eyes, separated from eye margins by less than half of their own diameter. Mouthparts light brownish. Palpus with basal three segments brown, $4^{\text {th }}$ segment light brown, $5^{\text {th }}$ segment yellowish. Scape dark brown, pedicel and all flagellomeres somewhat lighter. Flagellomeres cylindrical, about two times as long as broad.

Thorax. All parts of thorax blackish brown to black, with light setae. Scutum somewhat darker than lateral parts of thorax. Scutellum with numerous setae, marginal setae not arranged to distinct pairs. Antepronotum and proepisternum with pale bristles and short darker setae. Laterotergite and other pleural parts bare. Haltere pale yellow.

Legs. All coxae, trochanters, femora and tibiae dark brown. Tibial spurs yellow. Tarsi dark yellow but seem brownish because of dense setae. Fore tibia apicomedially with semicircular tibial organ (anteroapical depressed area), without strong setae, only densely covered with fine yellow setulae.

WINGS. Hyaline, unmarked. Radial veins and r-m dark brown, other veins paler, $\mathrm{m}$-stem faint. Sc, Rs, $\mathrm{m}$-stem, basal part of $\mathrm{M}_{1}, \mathrm{bm}-\mathrm{m}$ and basal one third of cu-stem asetose, other veins setose. Costa reaches to about one third of distance between $\mathrm{R}_{5}$ and $\mathrm{M}_{1}$. Sc ends in $\mathrm{R}$ at level of beginning of $\mathrm{m}$-stem. Posterior fork begins well before anterior fork, approximately at level of basal fifth of $r-m$.

AвDOMEn. Dark brown with pale setae, first three segments with tergites laterally and sternites wholly yellowish.

Terminalia (Figs 3A-E, 7B). Brown, with gonostyli and posteroventral area of gonocoxites yellow. Tergite 9 subquadrate, about as long as broad, posterior margin straight, with submarginal row of prominent setae about twice as long as other vestiture on tergite. Posteroventral margin of gonocoxites with quadrate, posteriorly slightly widening medial process having lateral aggregations of dark setae, medially separated by stripe of light setae. Gonostylus simple, tapering, apical half bent medially, with (1) long and pointed medial spine at medial margin, (2) row of three more anterior, blunt spines at 
medial margin, about three times shorter than posterior spine, (3) medial surface bearing minute setae, and (4) lateral surface bearing about 15 fine setae medially. Cercus with 13 combs of retinacula.

\section{Female}

Unknown.

\section{Biology}

Unknown.

Docosia peloponnensis Ševčík sp. nov. urn:1sid:zoobank.org:act:979DF75A-E677-4C63-8B13-BB3F3270115F

Figs 4, 7D

\section{Differential diagnosis}

This species is unique among the species of Docosia in having a simple gonostylus with a sickle-like posterior projection.

\section{Etymology}

The species name refers to the type locality.

\section{Type material}

\section{Holotype}

GREECE • Ō; Peloponnese, Valtesiniko env.; 1100 m a.s.l.; 22 Apr. 2016; J. Ševčík leg.; sweeping along small brook; in a pinned microvial with glycerol; specimen after DNA extraction; GenBank: MT293011, MT293042, MT293059, MT292993; NMPC- JSDO15.

\section{Paratype}

GREECE • 1 đ̊ં; Peloponnese, Valtesiniko env.; 1100 m a.s.1.; 26 Apr. 2016; M. Tkoč leg.; sweeping along small brook; in a pinned microvial with glycerol; JSL-UOC-JSDO15b.

\section{Description}

Male $(n=2)$

MEASUREMENTS. Length of wing $4.2 \mathrm{~mm}$.

HEAD. Blackish brown with numerous pale setae. Three ocelli, lateral ones almost touching compound eyes, separated from eye margins by less than half of their own diameter. Clypeus blackish, with setae pale. Mouthparts light brownish. Palpus brownish yellow, basal segment and apical part of last palpomere darker. Scape, pedicel and all flagellomeres dark brown. Flagellomeres cylindrical, flagellomeres 1 to 7 about twice as long as broad, distal flagellomeres ( 8 to 14 ) slightly conical, 2.5 to 3 times as long as broad.

THORAX. All parts of thorax blackish brown, with light setae. Scutellum with numerous setae and several submarginal pale bristles about twice as long as scutellum. Antepronotum and proepisternum with pale bristles and short darker setae. Laterotergite and other pleural parts bare. Haltere pale yellow.

LEGS. All coxae yellow, with basal third darkened. Femora mostly yellow, fore and mid femur dark along ventral margin, more extensively in proximal third; similarly hind femur, brownish also around tip. All trochanters blackish brown. Tibiae and tarsi yellow, tarsal segments seemingly brownish because of dense setulae. Fore tibia apicomedially with semicircular tibial organ (anteroapical depressed area), without strong setae, only densely covered with fine setulae. 


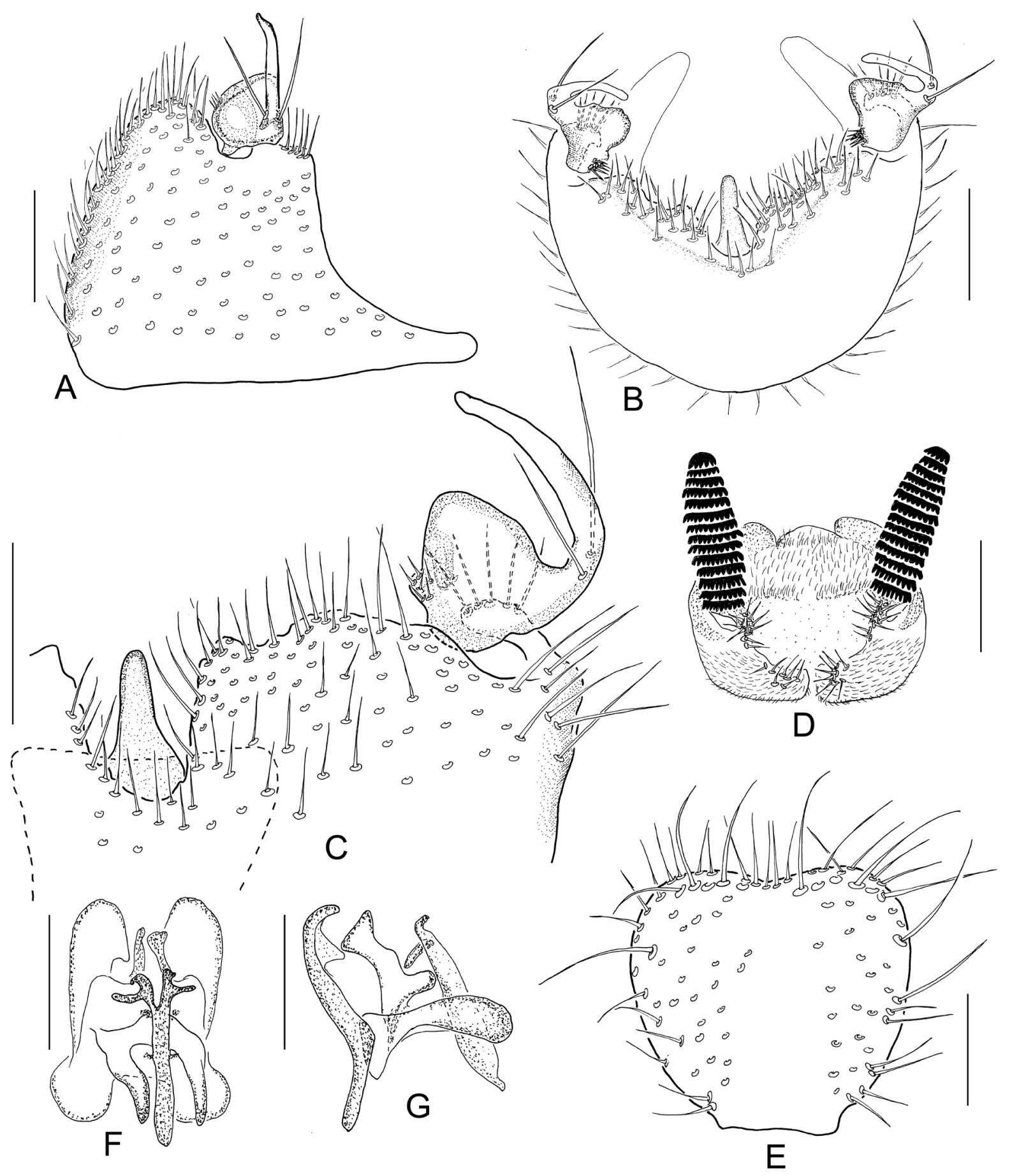

Fig. 4. Docosia peloponnensis Ševčík sp. nov., $\widehat{~}$, terminalia. A. Lateral view. B. Posterior view. C. Ventral view of gonostylus. D. Dorsal view of cerci. E. Dorsal view of tergite 9. F. Ventral view of aedeagal complex. G. Lateral view of aedeagal complex. Scale bars $=0.1 \mathrm{~mm}$. 
WINGS. Hyaline, unmarked. Radial veins and $\mathrm{r}-\mathrm{m}$ dark brown, other veins paler, basal parts of $\mathrm{m}$ faint, almost not traceable. Sc, Rs and basal third of cu-stem asetose, other veins setose. Costa reaches to 0.45 of distance between $R_{5}$ and $M_{1}$. Sc ends in R slightly beyond level of beginning of $\mathrm{m}$-stem. Posterior fork begins before anterior fork, approximately at level of basal third of r-m.

ABDomen. All dark brown.

Terminalia (Figs 4A-E, 7D). Dark brown except lighter gonostyli and posterior margin of gonocoxites. Tergite 9 about as long as broad, slightly narrowing towards base, with slightly concave posterior margin. Posteroventral margin of gonocoxites without lateral projections, with distinct U-shaped medial incision and narrow digitiform medioventral process. Gonostylus simple, slightly bulging medially, with aggregation of small setae at mediobasal corner and with characteristic sickle-like posterior projection, with prominent basal setae at its ventral and dorsal sides. Cercus with 14 combs of retinacula.

\section{Female}

Unknown.

\section{Biology}

Unknown.

\section{Docosia polyspina Kurina sp. nov. urn:1sid:zoobank.org:act:53D312AC-5DAB-4B0D-AF1C-819CAC59965F}

Figs $5,7 \mathrm{C}$

\section{Differential diagnosis}

Docosia polyspina sp. nov. belongs to a large group of Palaearctic species that have a simple, undivided gonostylus, bearing a variable number of black spines in different positions (e.g., D. flavicoxa Strobl, 1900, D. moravica Landrock, 1916, D. dentata Ševčík et al., 2016, D. matilei Ševčík \& Laštovka, 2008, D. kerkini, D. svanetica sp. nov., D. japonica sp. nov.). Docosia polyspina sp. nov. markedly differs from the other species in having the gonostylus with three long spines at the medial margin and the internal flange of the posteroventral margin of the gonocoxites with about 10 simple blunt spines laterally on both sides.

\section{Etymology}

The name is combined from the Greek ' $\pi$ o $\lambda v$ ' (polú) 'many' and Latin 'spina' 'spine', referring to the number of black spines on internal flange of the gonocoxites posteroventrally.

\section{Type material}

\section{Holotype}

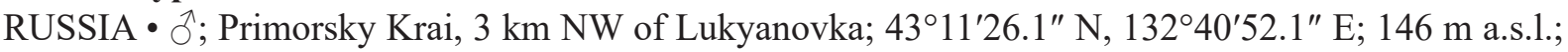
9 May 2019; O. Kurina leg.; sweep net; mounted from ethyl alcohol; terminalia in glycerol; one fore leg used for DNA extraction; GenBank: MT293025, MT293056, MT293039, MT293073, MT293007; IZBE-0200401.

\section{Description}

$$
\text { Male }(\mathrm{n}=1)
$$

MEASUREMENTS. Length of wing $3.0 \mathrm{~mm}$.

HEAD. Blackish with numerous pale setae. Three ocelli, lateral ones almost touching compound eyes, separated from eye margins by less than half of their own diameter. Mouthparts yellow to light brownish. 

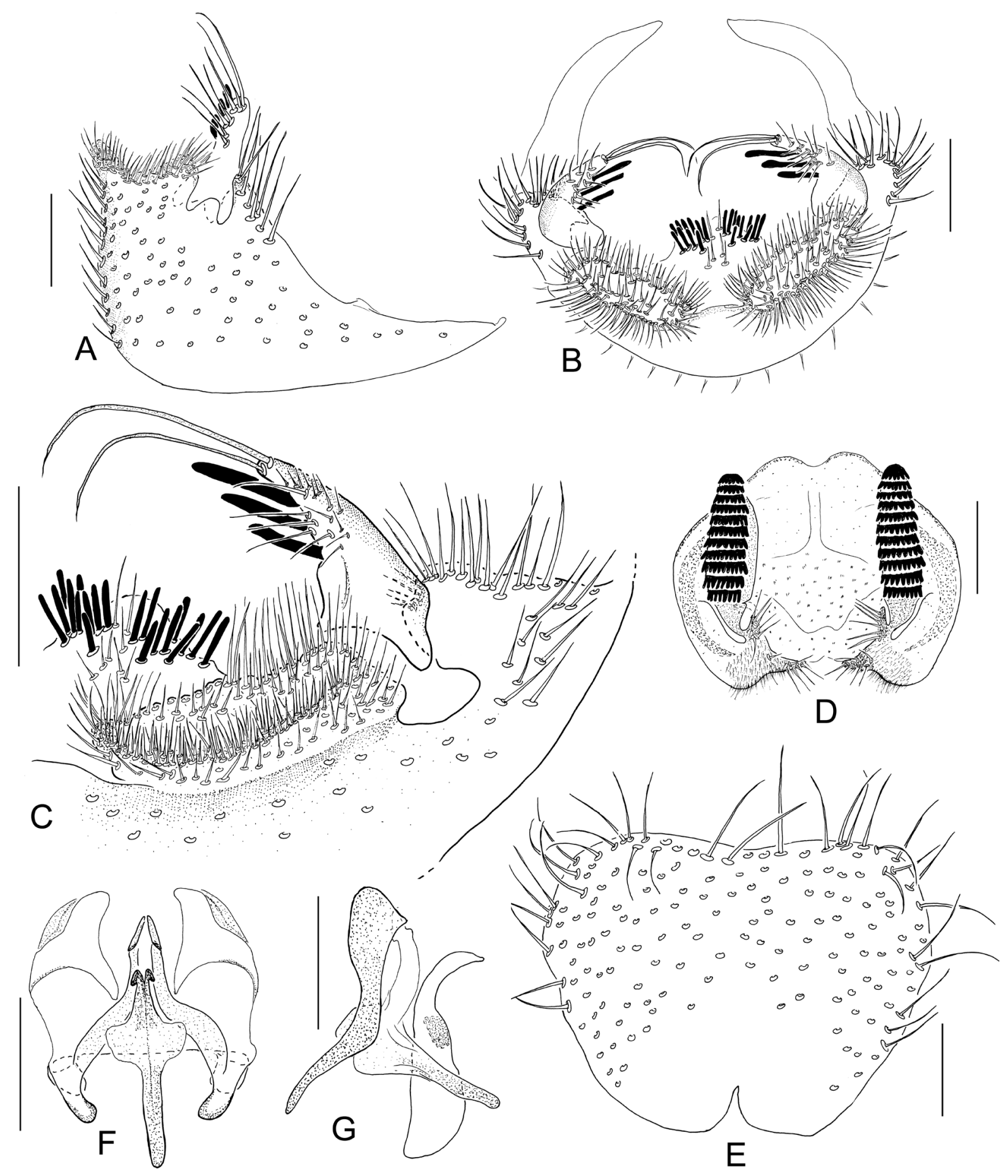

Fig. 5. Docosia polyspina Kurina sp. nov., ô, terminalia. A. Lateral view. B. Posterior view. C. Ventral view of gonostylus. D. Dorsal view of cerci. E. Dorsal view of tergite 9. F. Ventral view of aedeagal complex. G. Lateral view of aedeagal complex. Scale bars $=0.1 \mathrm{~mm}$. 
Palpus yellowish, basal segment somewhat darker. Scape dark brown, pedicel and basal half of first flagellomere dark yellow, rest of flagellomeres brown, lighter than scape. First three flagellomeres cylindrical, about 1.5 times as long as broad, rest of flagellomeres moniliform, about as long as broad.

THORAX. All parts of thorax blackish brown, with light setae. Scutellum with numerous setae and pair of submarginal pale bristles about twice as long as scutellum. Antepronotum and proepisternum with pale bristles and short darker setae. Laterotergite and other pleural parts bare. Haltere pale yellow.

LEGS. All coxae yellow, with basal fifth of fore- and midcoxae, and basal third of hind coxa darkened. Trochanters brownish. All femora and tibiae yellow. Fore tibia apicomedially with semicircular tibial organ (anteroapical depressed area), without strong setae, only densely covered with fine setulae. Tarsi seem brownish because of dense setae.

WiNGs. Hyaline, unmarked. Radial veins and r-m dark brown, other veins paler, m-stem faint, almost not traceable. Sc, Rs, m-stem, bm-m and basal two thirds of cu-stem asetose, other veins setose. Costa reaches to about one third of distance between $R_{5}$ and $M_{1}$. Sc ends in $R$ at level of beginning of $m$-stem. Posterior fork begins well before anterior fork, approximately at level of basal fifth of r-m.

Abdomen. All dark brown with pale setae.

Terminalia (Figs 5A-E, 7C). Light brown, with gonostyli slightly lighter. Tergite 9 widening posteriorly, about 1.3 times as long as broad, posterior margin slightly concave, anterior margin with V-shaped medial cleft. Posteroventral margin of gonocoxites with lateral extensions, covered with dense aggregation of fine setae, setae longer at posterior margin. Internal flange of posteroventral margin drawn out to large, posteriorly rounded medial process, with about 10 simple blunt spines laterally on both sides and about 10 setae medially. Gonostylus simple, tapering, with (1) row of three blunt medially directed spines at medial margin, about twice as long as spines on internal flange of posteroventral margin of gonocoxites, (2) two extremely long apically curved setae, clearly deviating from other setae of terminalia, and (3) about 15 setae on apical half ventrally. Cercus with 11 combs of retinacula.

\section{Female}

Unknown.

\section{Biology}

Unknown.

Docosia svanetica Kurina sp. nov.

urn:1sid:zoobank.org:act:51A54E2C-274B-4DB9-880B-0FDF2020E34A

Figs 1, 6, 7E

\section{Differential diagnosis}

By the structure of the male terminalia, D. svanetica sp. nov. belongs to a group of Palaearctic species as defined for $D$. polyspina sp. nov. and resembles D. kerkini. Both species have a simple sickle- or crescent-shaped gonostylus with a row of medial spines and a posteroventral margin of gonocoxites with submedial humps, bearing a comb of short setae. The subsimilar combs of the setae at the posteroventral margins of the gonocoxites are represented in several Palaearctic species (e.g., D. dentata, D. lastovkai Chandler, 1994, D. melita Chandler et al., 2006), which have an otherwise different structure of the gonostylus and aedeagal complex. Dcosia svanetica sp. nov. differs markedly from $D$. kerkini in the shape of the ninth tergite, which is subcircular, while it is apically very wide and broadly concave in D. kerkini. Moreover, D. svanetica sp. nov. has a gonostylus with a prominent apical and four smaller 
medial spines in spite of about 12 medial subequal spines in D. kerkini. The laterotergite is setose in D. svanetica, while it is bare in D. kerkini.

\section{Etymology}

The species name refers to the type locality in Svanetia, a historic province of Georgia, in the northwestern part of the country.

\section{Type material}

\section{Holotype}

GEORGIA - ${ }^{\top}$; Samegrelo-Zemo Svaneti, near Ushguli, path to glacier; $42^{\circ} 56^{\prime} 37.4^{\prime \prime} \mathrm{N}, 43^{\circ} 3^{\prime} 14.2^{\prime \prime} \mathrm{E}$; 2220 m a.s.l.; 15-17 Jun. 2019; X. Mengual leg.; Malaise trap; in ethyl alcohol; ZFMK-DIP-00067332.

\section{Paratypes}

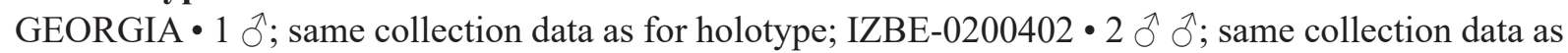

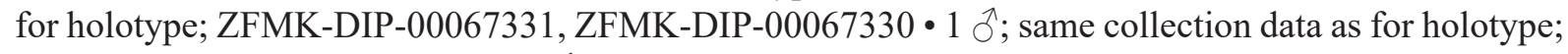
IUTG ZFMK-DIP-00067329 - 1 ; ; same collection data as for holotype; after DNA extraction; GenBank: MT293024, MT293055, MT293038, MT293072, MT293006; JSL-UOC-JSDO39 • 1 §;; Samegrelo-Zemo Svaneti, S of Lakhushdi, meadow; 42 ${ }^{\circ} 59^{\prime} 55.8^{\prime \prime}$ N, 42 ${ }^{\circ} 39^{\prime} 00^{\prime \prime}$ E; 1270 m a.s.1.; $13-14$ Jun. 2019; X. Mengual leg.; Malaise trap; in alcohol; IZBE-0200403 • 1 §ं; Samegrelo-Zemo Svaneti, S of Lakhushdi, meadow; 42 59'56.3" N, 42³9'07" E; 1270 m a.s.1.; 13-14 Jun. 2019; X. Mengual leg.; double Malaise trap; in alcohol; IZBE-0200404 - 1 \% ; Samtskhe-Javakheti, road from Abastumani to

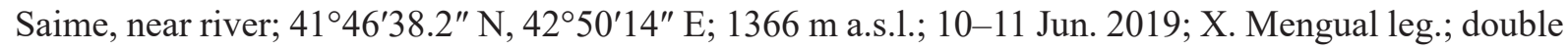
Malaise trap; in alcohol with terminalia in glycerol; IZBE-0200405.

\section{Description}

Male $(n=9)$

MeAsurements. Length of wing 3.0-3.4 $\mathrm{mm}$ (holotype $=3.2 \mathrm{~mm}$ ).

HEAD. Blackish brown with numerous pale setae. Three ocelli, the lateral ones almost touching compound eyes, separated from eye margins by less than half of their own diameter. Mouthparts light brownish. Palpus with two basal segments brownish and three apical segments yellow. Scape, pedicel and all flagellomeres dark brown. Flagellomeres cylindrical, flagellomeres 1 and 2 about 1.3 times as long as broad, other flagellomeres $2-2.5$ times as long as broad.

THORAX. All parts of thorax dark brown to blackish, with light setae. Scutellum with numerous pale setae and several submarginal pale bristles about twice as long as scutellum. Antepronotum and proepisternum with pale bristles and short darker setae. Laterotergite with 6-8 light bristles on posterior half, other pleural parts bare. Haltere pale yellow.

LEGS. All coxae yellow, with basal fifth of fore- and midcoxae, and basal third of hind coxa darkened. Trochanters brownish. Femora mostly yellow, fore and mid femora dark along ventral margin in proximal half, hind femur apically darkened. All tibiae yellow. Fore tibia apicomedially with semicircular tibial organ (anteroapical depressed area), without strong setae, only densely covered with fine setulae. Tarsi seem darker because of dense setae.

WiNGs. Hyaline, unmarked. Radial veins and $\mathrm{r}-\mathrm{m}$ dark brown, other veins paler, $\mathrm{m}$-stem and basal parts of $M_{1}$ and $M_{2}$ faint, almost not traceable. Sc, Rs, bm-m, m-stem and basal third of cu-stem asetose, other veins setose. Costa reaches to about one third of the distance between $R_{5}$ and $M_{1}$. Sc ends in $R$ at level of beginning of m-stem. Posterior fork begins before anterior fork, approximately at level of basal third of r-m. 
AвDOMEN. Dark brown to blackish, with second and third segments light brown.

Terminalia (Figs 6A-E, 7E). Dark brown except lighter gonostyli. Tergite 9 subcircular, about as long as broad, posterior margin slightly concave, anterior margin with wide V-shaped incision medially.

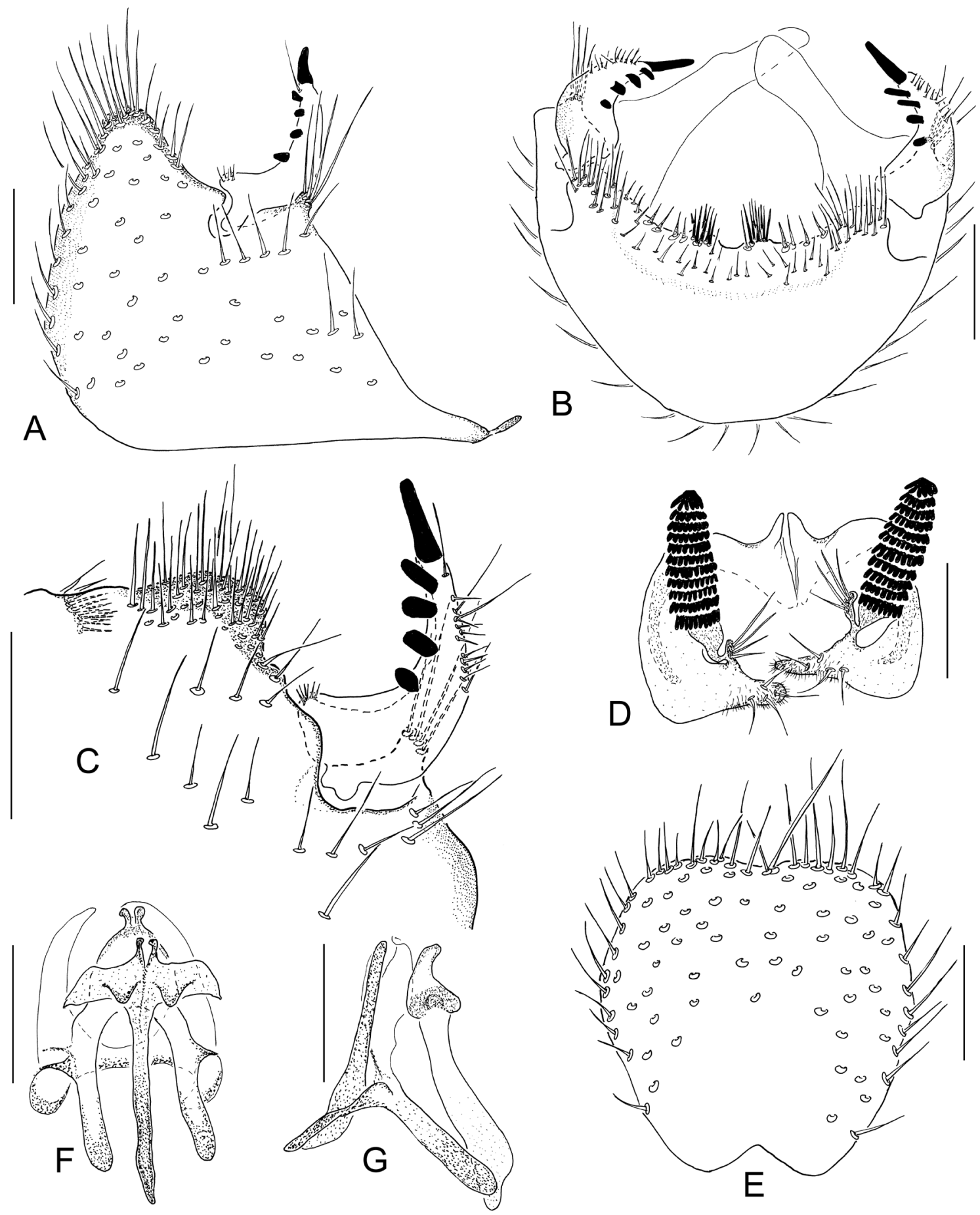

Fig. 6. Docosia svanetica Kurina sp. nov., ภ̂, terminalia. A. Lateral view. B. Posterior view. C. Ventral view of gonostylus. D. Dorsal view of cerci. E. Dorsal view of tergite 9 . F. Ventral view of aedeagal complex. G. Lateral view of aedeagal complex. Scale bars $=0.1 \mathrm{~mm}$. 
Posteroventral margin of gonocoxites with extended flange, with submedial humps bearing combs of strong short setae. Gonostylus simple, sickle-shaped, tapering, with (1) apical blunt spine, (2) row of four blunt spines medially, about twice shorter than the apical one, and (3) about 15 setae laterally on apical half. Cercus with 11 combs of retinacula.
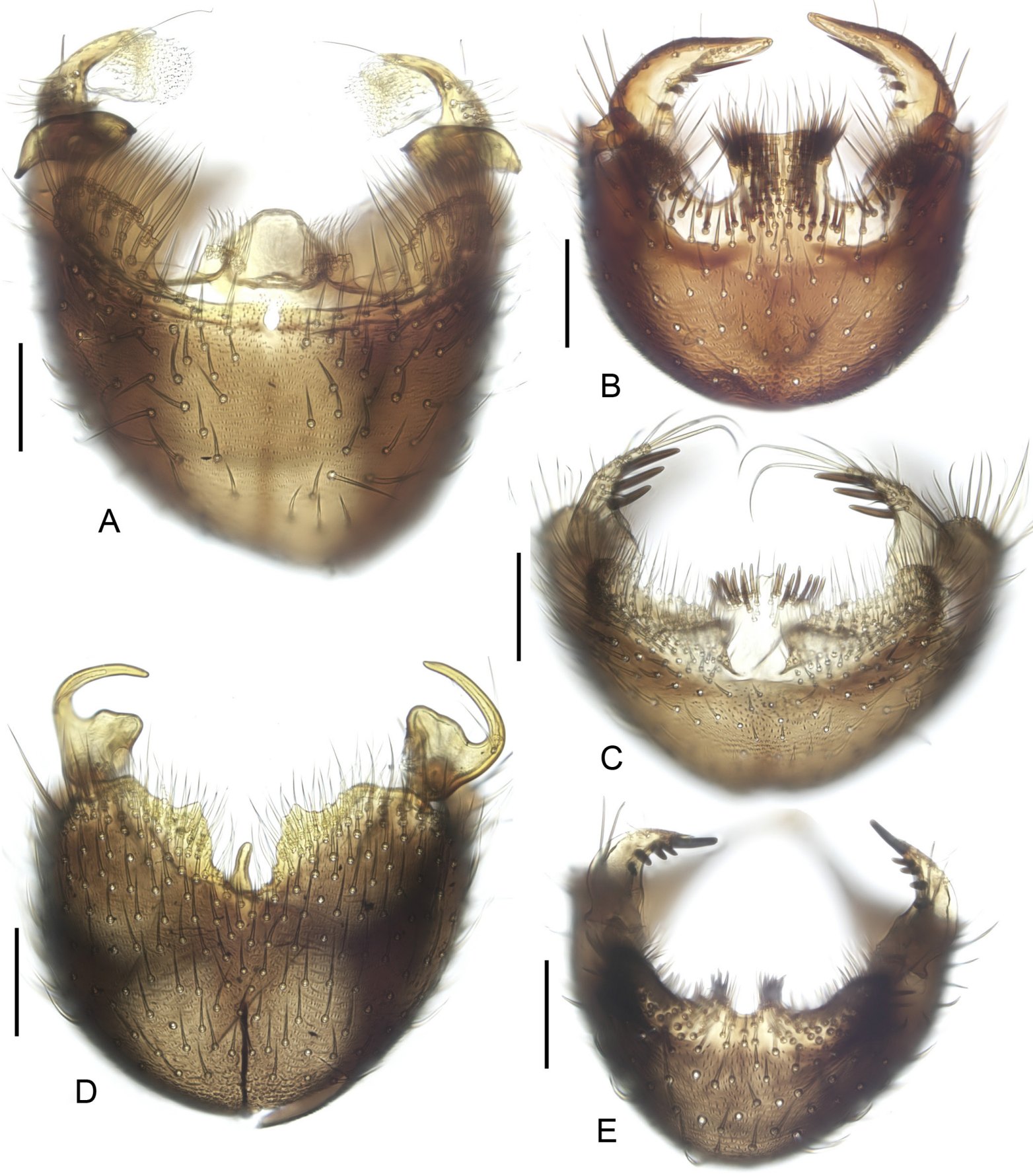

Fig. 7. Male terminalia, ventroposterior view. A. Docosia anatolica Ševčík sp. nov. B. D. japonica Kurina sp. nov. C. D. polyspina Kurina sp. nov. D. D. peloponnensis Ševčík sp. nov. E. D. svanetica Kurina sp. nov. Scale bars $=0.1 \mathrm{~mm}$. 


\section{Female}

Unknown.

\section{Biology}

Unknown.

\section{Molecular phylogeny of Docosia}

The phylogenetic tree for the concatenated dataset based on five gene fragments is presented in Fig. 8 . The genus Docosia was found to be monophyletic with a maximum ultrafast bootstrap support value (ufboot $=100$ ).

A clade containing two species, D. fumosa Edwards, 1925 and D. morionella Mik, 1884, branched most basally, as a sister group to all the other species of Docosia included in this analysis, followed by two species of the D. gilvipes (Walker, 1856) group and two species of the D. dichroa Loew, 1869 group. All the other species of Docosia still form a monophyletic group, though with moderate support (ufboot = 93). Within this group, the following pairs of species grouped together: D. carbonaria Edwards, 1941 + D. rameli Kurina \& Ševčík, 2011 (ufboot $=100$ ), followed by D. cephaloniae Chandler, Bechev \& Caspers, $2006+$ D. lastovkai (ufboot $=100)$ and D. muranica Kurina \& Ševčík, $2011+$ D. fuscipes (Roser, 1840), ufboot $=93$.

Most of the relationships among the rest of the species included to this dataset are less supported (ufboot $<80$ ), except for the group of six species related to D. nigra Landrock, 1928, which apparently constitute a monophyletic group ( $\mathrm{ufboot}=85$ ).

\section{New national records}

The opportunity is taken here to record the following species as new to regional faunas.

\section{Docosia diutina Plassmann, 1996}

\section{Material examined}

TURKEY • 1 O; Mugla, 11 km east, pine wood and meadow; 1310 m a.s.1.; 1 May 2013; M. Barták and Š. Kubík leg.; in ethanol; JSL-OUC-DOD.

\section{Material examined}

Docosia flavicoxa Strobl, 1900

GEORGIA • 3 $\widehat{\partial}$; Lagodekhi Nature Reserve, Matsimi river gorge; 17-27 Jun. 2011; G. Japoshvili leg.; Malaise trap; in ethanol; IZBE-0200406.

\section{Docosia gilvipes (Haliday in Walker 1856)}

\section{Material examined}

GEORGIA • 1 \%; Marelisi southeast of Surami; 41 ${ }^{\circ} 57^{\prime} 56^{\prime \prime}$ N, 4317'20.7" E; 412 m a.s.1.; 19 May 2012; O. Kurina leg.; sweep net; in ethanol; IZBE-0200407 • 1 đ'; Samegrelo-Zemo Svaneti, S of Lakhushdi, meadow; 4259'56.3" N, 42³9'0.7" E; 1270 m a.s.1.; 13-14 Jun. 2019; X. Mengual leg.; double Malaise trap; in ethanol; ZFMK-DIP-00067328. 
Docosia kerkini Kurina \& Ševčík, 2011

\section{Material examined}

BULGARIA • 1 ठ̊; Pavel Banya, 6 km S; 5-6 May 2018; M. Barták and Š. Kubík leg.; sweep net; in ethanol; JSL-OUC- JSDO34.

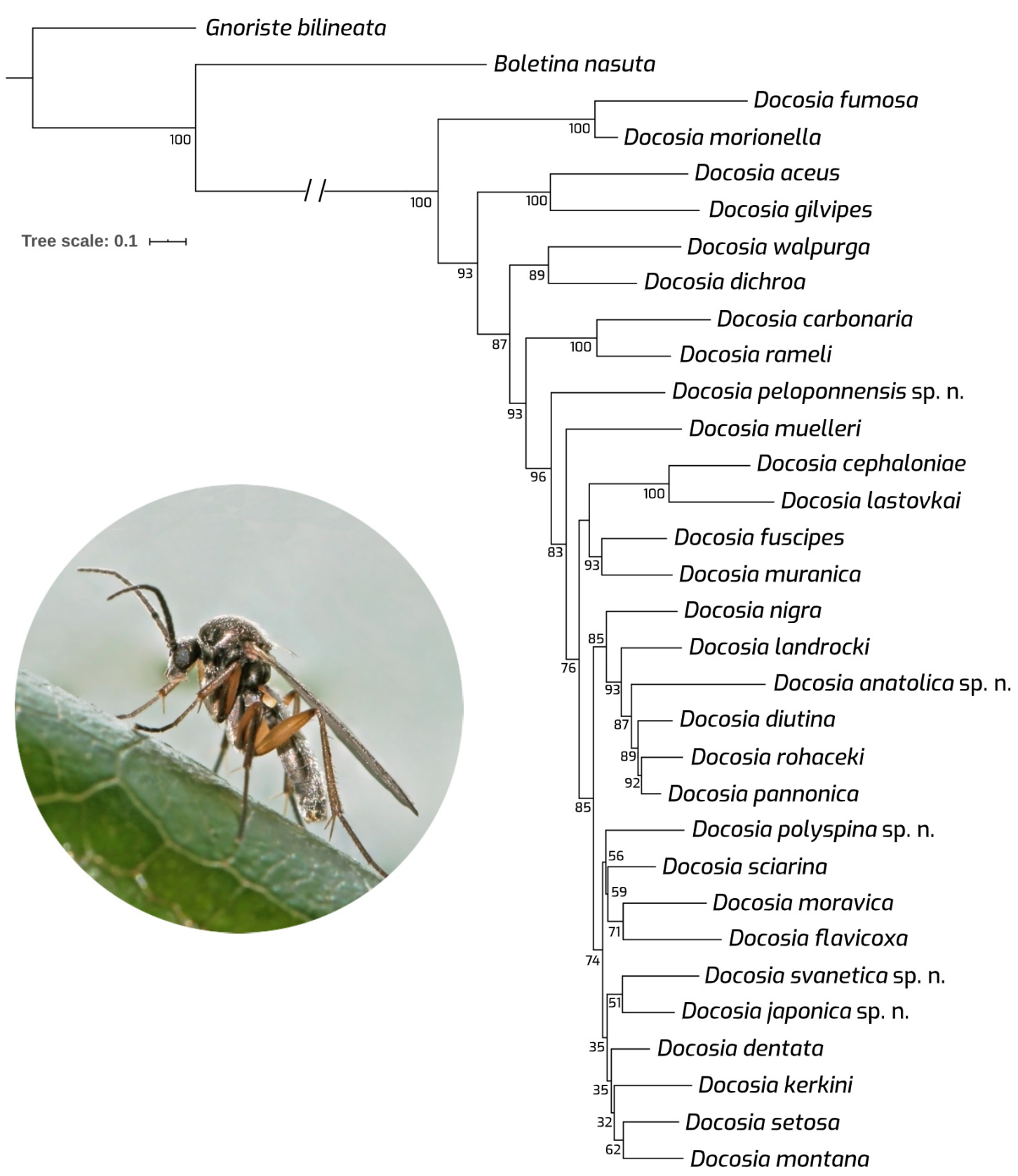

Fig. 8. Maximum likelihood hypothesis for relationships among selected species of Docosia Winnertz, 1863 based on DNA sequence data (28S, ITS2, COI, COII and CytB), 3049 characters. Above node number $=$ ultrafast bootstrap values (ufboot). The stem of the Docosia clade has been shortened to half its original length. 
ŠEVČÍK J. et al., New species and updated phylogeny of Docosia

Docosia moravica Landrock, 1916

\section{Material examined}

GEORGIA • 1 ơ $^{\lambda}$ S Samtskhe-Javakheti, road from Abastumani to Saime; $41^{\circ} 47^{\prime} 48.8^{\prime \prime}$ N, $42^{\circ} 50^{\prime} 38^{\prime \prime}$ E; 1725 m a.s.1.; 10-11 May 2019; X. Mengual leg.; Malaise trap; in ethanol; ZFMK-DIP-00067327.

Docosia pannonica Laštovka \& Ševčík, 2006

\section{Material examined}

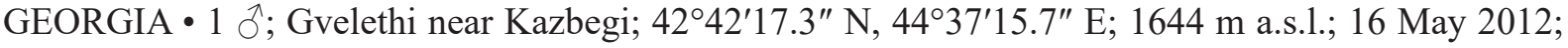
O. Kurina leg.; light trap; in ethanol; IZBE-0200408.

\section{Docosia rameli Kurina \& Ševčík, 2011}

\section{Material examined}

SLOVAKIA • 1 o'; Horša, Horšianska dolina; 48 $18^{\prime} 07.88^{\prime \prime}$ N, $18^{\circ} 41^{\prime} 57.30^{\prime \prime}$ E; 230 m a.s.1.; 26 Apr.5 May 2017; L'. Vidlička and O. Majzlan leg.; Malaise trap; in ethanol; JSL-OUC- JSDO29.

\section{Discussion}

If we compare the tree in Fig. 8 with the previous molecular phylogeny of Docosia by Ševčík et al. (2016a), it is evident that more extensive taxon sampling and the addition of one gene fragment have not increased the resolution substantially. Although we used a different algorithm (IQ-Tree vs RAxML in the previous paper), the node support values are in both cases relatively low, indicating that new and more variable molecular markers should be tested to recover the interrelationships among particular species of Docosia.

Several pairs of closely related species can be recognized in the molecular tree (Fig. 8), although a close relationship between relevant species in a pair is not always clear from a morphological point of view. The first example is the well-supported clade comprising Docosia fumosa and D. morionella. Although the former species is widely distributed, relatively well known, and its terminalia were figured, e.g., by Laštovka \& Ševčík (2006), the latter species is extremely rare, with only several records available and with male terminalia figured by Chandler \& Blasco-Zumeta (2001). It is difficult to find distinct similarities between these two species. The laterotergite is pubescent in D. fumosa but bare in D. morionella, showing that pubescence of laterotergite is apparently a homoplastic character, evolved in parallel in different lineages (see also Ševčík et al. 2016a). Concerning the colouration of the legs, both D. fumosa and $D$. morionella have a black hind femur, but this character state is also present in D. carbonaria and some other species. On the other hand, the male terminalia of $D$. morionella are rather similar to those of $D$. gilvipes, especially in the overall shape of the gonocoxites and the structure of the cerci, as noted by Chandler \& Blasco-Zumeta (2001). However, D. gilvipes differs in having Sc setose and ending free. The latter character has been widely used to separate $D$. gilvipes from the other Palaearctic species. However, another very similar species, D. pseudogilvipes Kurina, 2008 recorded from the Italian Alps, has Sc bare and ending in R (Kurina 2008). Morover, other species considered as related to D. gilvipes, e.g., the Nearctic D. aceus Garrett, 1925 (and probably also D. mcgrawi Taber, 2018) have Sc ending in $\mathrm{R}$, while the recently described Neotropical species D. adusta Oliveira \& Amorim, 2011 has Sc ending free and bare (Oliveira \& Amorim 2011). Such a variation in wing venation shows that morphological characters other than on male terminalia should be used with caution in phylogenetic reconstructions of Docosia, and possibly also within other genera of fungus gnats. However, a careful case-by-case study is required before any general conclusion can be drawn. 
Two species of the $D$. dichroa Loew, 1869 group are easily recognizable thanks to their rufous abdomen, a unique feature among all species of Docosia. We follow here the interpretation of these two species by Taber $(2011,2012)$, although Petr Laštovka in his unpublished material (original figures plus handmade notes available to the first author) came to the conclusion that the species recently named by Taber (2011) as D. walpurga is actually true $D$. dichroa, and D. dichroa sensu Johannsen (1909) and Taber $(2011,2012)$ needs a new name. However, we leave this issue here as open, until a re-examination of the type material is made (see also discussion in Taber 2018).

Another well-supported clade is $D$. carbonaria $+D$. rameli. Both species share the pubescence of the laterotergites, the similar shape of gonostylus and the overall structure of the posterior margin of the gonocoxites. Also, D. cephaloniae and D. lastovkai share many synapomorphies, mainly on the male terminalia, and several other species belong to this group of species as well (cf. Chandler et al. 2006). Both D. carbonaria and D. lastovkai apparently represent complexes of closely related species, especially in the Mediterranean region and eastern Europe, and other species may be discovered in the future.

Three southeastern European species (D. diutina, D. pannonica and D. rohaceki) represent a specific and well-defined group, based on their male terminalia. Especially the longitudinal tergite 9, distinct posterolateral processes of gonocoxites and the apically modified (ramifying) setae at the posteroventral margin of the gonocoxites are very characteristic. These species also share some colour characteristics, e.g., a dark basal half of the hind coxa. Most of these features are also present in D. anatolica sp. nov. and D. nigra. All these species constitute a monophyletic group in the molecular tree (Fig. 8). In addition, several species not included in our molecular analysis certainly belong to this group, e.g., D. juxtamontana Chandler, 2004.

\section{Acknowledgements}

We are grateful to Miroslav Barták (Prague, Czech Republic), Scott Fitzgerald (Oregon, USA), Milan Kozánek (Bratislava, Slovakia), Jindřich Roháček (Opava, Czech Republic), Ximo Mengual (Bonn, Germany) and Stephen Taber (Saginaw Valley State University, USA) for providing specimens for this study. The study was supported by the Ministry of Culture of the Czech Republic by institutional financing of long-term conceptual development of the research institution (the Silesian Museum, MK000100595), and by the internal grants for specific research at the University of Ostrava (SGS10/

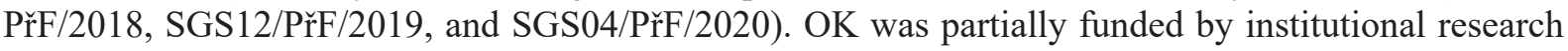
funding (IUT21-1) of the Estonian Ministry of Education and Research and by European Commission's Research Infrastructure programme SYNTHESYS+ (DE-TAF-2498). Peter Chandler and Eirik Rindal provided useful comments and suggestions to the text.

\section{References}

Chandler P.J. 2010. A Dipterist's Handbook. Vol. 15. $2^{\text {nd }}$ Ed. The Amateur Entomologists' Society, London.

Chandler P.J. \& Blasco-Zumeta J. 2001. The fungus gnats (Diptera, Bolitophilidae, Keroplatidae and Mycetophilidae) of the Monegros Region (Zaragoza, Spain) and five other new species of Pyratula Edwards and Sciophila Meigen. Zapateri, Revista aragonesa de Entomologia 9: 1-24.

Chandler P.J., Bechev D.N. \& Caspers N. 2006. The fungus gnats (Diptera: Bolitophilidae, Diadocidiidae, Ditomyiidae, Keroplatidae and Mycetophilidae) of Greece, its islands and Cyprus. Studia Dipterologica 12 (2005): 255-314.

Hoang D.T., Chernomor O., von Haeseler A., Minh B.Q. \& Vinh L.S. 2017. UFBoot2: Improving the ultrafast bootstrap approximation. Molecular Biology and Evolution 35: 512-522.

https://doi.org/10.1093/molbev/msx281 
ŠEVČÍK J. et al., New species and updated phylogeny of Docosia

Jakovlev J. 2012. Fungal hosts of mycetophilids (Diptera: Sciaroidea excluding Sciaridae): a review. Mycology 3: 11-23.

Johannsen O.A. 1909. Diptera. Fam. Mycetophilidae. In: Wytsman P. (ed.) Genera Insectorum 93: $1-141$. Brussels.

Jürgenstein S., Kurina O. \& Põldmaa K. 2015. The Mycetophila ruficollis Meigen (Diptera, Mycetophilidae) group in Europe: elucidating species delimitation with COI and ITS2 sequence data. ZooKeys 508: 15-51. https://doi.org/10.3897/zookeys.508.9814

Kaspřák D., Kerr P., Sýkora V., Tóthová A. \& Ševčík J. 2019. Molecular phylogeny of the fungus gnat subfamilies Gnoristinae and Mycomyinae, and their position within Mycetophilidae (Diptera). Systematic Entomology 44: 128-138. https://doi.org/10.1111/syen.12312

Kurina O. 2006. Three new species of Docosia Winnertz (Diptera: Mycetophilidae) from Kazakhstan. Entomologica Fennica 17 : 110-117. https://doi.org/10.33338/ef.84296

Kurina O. 2008. Sciaroidea excl. Sciaridae. In: Ziegler J. (ed.) Diptera Stelviana. A dipterological perspective on a changing alpine landscape. Vol. 1. Studia Dipterologica Supplements 16: 245-293.

Kurina O. \& Ševčík J. 2011. Three new species of Docosia Winnertz from central and southern Europe (Diptera: Mycetophilidae). Zootaxa 2810: 26-36. https://doi.org/10.11646/zootaxa.2810.1.3

Kurina O. \& Ševč́́k J. 2012. Notes on Docosia Winnertz (Diptera: Mycetophilidae), with description of six new species from Central Asia and the first generic record from the Afrotropical region. Zootaxa 3570: 25-40. https://doi.org/10.11646/zootaxa.3570.1.2

Laštovka P. \& Ševčík J. 2006. A review of the Czech and Slovak species of Docosia Winnertz (Diptera: Mycetophilidae), with atlas of the male and female terminalia. Ćasopis Slezského zemského muzea Opava (A) 55: 1-37.

Letunic I. \& Bork P. 2016. Interactive tree of life (iTOL) v3: An online tool for the display and annotation of phylogenetic and other trees. Nucleic Acids Research 44: 242-245.

https://doi.org/10.1093/nar/gkw290

Minh B.Q., Nguyen M.A.T. \& von Haeseler A. 2013. Ultrafast approximation for phylogenetic bootstrap. Molecular Biology and Evolution 30: 1188-1195. https://doi.org/10.1093/molbev/mst024

Nguyen L.T., Schmidt H.A., von Haeseler A. \& Minh B.Q. 2015. IQ-TREE: A fast and effective stochastic algorithm for estimating maximum likelihood phylogenies. Molecular Biology and Evolution 32: 268-274. https://doi.org/10.1093/molbev/msu300

Oliveira S.S. \& Amorim D.S. 2011. Docosia adusta sp. nov. (Diptera, Mycetophilidae) from the Colombian Andes: a Holarctic element in northwestern South America. Canadian Entomologist 143: 688-696. https://doi.org/10.4039/n11-033

Oliveira S.S. \& Amorim D.S. 2014. Catalogue of Neotropical Diptera. Mycetophilidae. Neotropical Diptera 25: 1-87.

Rindal E., Søli G.E.E. \& Bachmann L. 2009. Molecular phylogeny of the fungus gnat family Mycetophilidae (Diptera, Mycetophiliformia). Systematic Entomology 34: 524-532.

https://doi.org/10.1111/j.1365-3113.2009.00474.x

Rulik B. \& Kallweit U. 2006. A blackbird's nest as breeding substrate for insects - first record of Docosia fumosa Edwards, 1925 (Diptera: Mycetophilidae) from Germany. Studia Dipterologica 13: 41-43.

Ševčík J. 2010a. Czech and Slovak Diptera Associated with Fungi. Slezské zemské muzeum, Opava.

Ševč́k J. 2010b. Docosia heikkii sp. nov., the first Oriental record of Docosia (Diptera: Mycetophilidae). Oriental Insects 44: 91-94. https://doi.org/10.1080/00305316.2010.10417609 
Ševčík J. \& Laštovka P. 2008. Two new European species of Docosia (Diptera: Mycetophilidae). Biologia 63: 117-119. https://doi.org/10.2478/s11756-008-0010-6

Ševčík J., Kaspřák D. \& TóthováA. 2013. Molecular phylogeny of fungus gnats (Diptera: Mycetophilidae) revisited: position of Manotinae, Metanepsiini, and other enigmatic taxa as inferred from multigene analysis. Systematic Entomology 38: 654-660. https://doi.org/10.1111/syen.12023

Ševčík J., Kaspřák D. \& Rulik B. 2016a. A new species of Docosia Winnertz from Central Europe, with DNA barcoding based on four gene markers (Diptera, Mycetophilidae). ZooKeys 549: 127-143.

https://doi.org/10.3897/zookeys.549.6925

Ševčík J., Kaspřák D., Mantič M., Fitzgerald S., Ševčíková T., Tóthová A. \& Jaschhof M. 2016b. Molecular phylogeny of the megadiverse insect infraorder Bibionomorpha sensu lato (Diptera). PeerJ 4: e2563. https://doi.org/10.7717/peerj.2563

Søli G.E.E. 1997. The adult morphology of Mycetophilidae (s. str.), with a tentative phylogeny of the family (Diptera, Sciaroidea). Entomologica Scandinavica Supplement 50: 1-55.

Søli G.E.E. 2017. 20. Mycetophilidae (Fungus Gnats). In: Kirk-Spriggs A.H. \& Sinclair B.J. (eds) Manual of Afrotropical Diptera. Vol. 2 (Nematocera \& Lower Brachycera). SANBI Publishing, Pretoria.

Stamatakis A. 2014. RAxML version 8: a tool for phylogenetic analysis and post-analysis of large phylogenies. Bioinformatics 30: 1312-1313. https://doi.org/10.1093/bioinformatics/btu033

Taber S.W. 2011. A New Species of Docosia Winnertz fungus gnat (Diptera: Mycetophilidae). Southwestern Entomologist 36: 451-464. https://doi.org/10.3958/059.036.0407

Taber S.W. 2012. A new Nearctic species of Docosia Winnertz fungus gnat (Diptera: Mycetophilidae), notes on Docosia walpurga Taber and Docosia dichroa Loew, and the identification of females of all three species. Southwestern Entomologist 37: 379-390. https://doi.org/10.3958/059.037.0314

Taber S.W. 2018. A new Nearctic species of Docosia Winnertz fungus gnat (Diptera: Mycetophilidae) from Michigan. Southwestern Entomologist 43: 683-689. https://doi.org/10.3958/059.043.0314

Trifinopoulos J., Nguyen L.T., von Haeseler A. \& Minh B.Q. 2016. W-IQ-TREE: a fast online phylogenetic tool for maximum likelihood analysis. Nucleic Acids Research 44: 232-235.

https://doi.org/10.1093/nar/gkw256

Vockeroth J.R. 1966. A method of mounting insects from alcohol. Canadian Entomologist 98: 69-70. https://doi.org/10.4039/Ent9869-1

Zaitzev A.I. 2011. Two new species of the genus Docosia Winnertz (Diptera: Mycetophilidae) from Russia and Turkmenistan. Russian Entomological Journal 20: 207-209.

https://doi.org/10.15298/rusentj.20.2.12

Manuscript received: 7 April 2020

Manuscript accepted: 27 July 2020

Published on: 17 September 2020

Topic editor: Nesrine Akkari

Desk editor: Radka Rosenbaumová

Printed versions of all papers are also deposited in the libraries of the institutes that are members of the EJT consortium: Muséum national d'histoire naturelle, Paris, France; Meise Botanic Garden, Belgium; Royal Museum for Central Africa, Tervuren, Belgium; Royal Belgian Institute of Natural Sciences, Brussels, Belgium; Natural History Museum of Denmark, Copenhagen, Denmark; Naturalis Biodiversity Center, Leiden, the Netherlands; Museo Nacional de Ciencias Naturales-CSIC, Madrid, Spain; Real Jardín Botánico de Madrid CSIC, Spain; Zoological Research Museum Alexander Koenig, Bonn, Germany; National Museum, Prague, Czech Republic. 